\title{
„Können wir es verantworten, ihn frei herumgehen zu lassen?"
}

\author{
Sexuelle Gewalt eines Klerikers im Feld von Theologie, Psychiatrie und Justiz \\ (1950er-1970er Jahre)
}

\author{
Christine Hartig
}

\section{Einleitung}

Die Analyse von einzelnen Fallgeschichten oder Fallstudien ${ }^{1}$ ist nicht mehr auf die zunächst klassischen Disziplinen der Rechtswissenschaft, Medizin und Psychologie beschränkt. ${ }^{2}$ Die sozialwissenschaftliche Forschung nutzt schon länger Einzelfälle, um soziales Handeln, häufig über den Weg der Typenbildung, zu reflektieren. ${ }^{3}$ Dieser Herangehensweise folgt in Teilen ebenso die MHG-Studie, die "spezifische Risikokonstellationen oder Vulnerabilitäten“4 bei Betroffenen und Tätertypen vorstellt. Auch einige von Jurist:innen erstellte Gutachten zum sexuellen Missbrauch von Klerikern in einzelnen Bistümern basieren auf einer Analyse von Fallgeschichten. Sie fokussieren zumeist die Frage nach der individuellen Verantwortung des Leitungspersonals der Diözesen. ${ }^{5}$ Mit der hier vorgelegten mikrohistorischen Fallgeschichte

1 Siehe zur Unterscheidung Ruben Hackler/Katherina Kinzel, Eine kleine Geschichte der Fallgeschichte, in: dies. (Hrsg.), Paradigmatische Fälle. Konstruktion, Narration und Verallgemeinerung von Fall-Wissen in den Geistes- und Sozialwissenschaften. Basel 2016, 5-26, hier 6.

2 Siehe beispielhaft Susanne Düwell/Nicolas Pethes, Fall, Wissen, Repräsentation - Epistemologie und Darstellungsästhetik von Fallnarrativen in den Wissenschaften vom Menschen, in: dies. (Hrsg.), Fall - Fallgeschichte - Fallstudie: Theorie und Geschichte einer Wissensform. Frankfurt a. M./New York 2014, 9-33, hier 9. Zur Genese von Fallgeschichten: Hackler/Kinzel, Fall.

3 Jürgen Fleiß, Paul Lazarsfelds typologische Methode und die Grounded Theory. Generierung und Qualität von Typologien, in: Österreichische Zeitschrift für Soziologie 35, 2010, 3-18.

4 Harald Dreßing u.a., Sexueller Missbrauch an Minderjährigen durch katholische Priester, Diakone und männliche Ordensangehörige im Bereich der Deutschen Bischofskonferenz (MHG-Studie), 2018, 67, einzusehen unter https:/www.dbk.de/fileadmin/redaktion/ diverse_downloads/dossiers_2018/MHG-Studie-gesamt.pdf, zuletzt aufgerufen am 24.2.2020.

5 Björn Gercke u.a., Gutachten. Pflichtverletzungen von Diözesanangehörigen des Erzbistums Köln im Umgang mit Fällen sexuellen Missbrauchs von Minderjährigen oder Schutzbefohlenen durch Kleriker oder sonstige pastorale Mitarbeitende des Erzbistums Köln im Zeitraum von 1975 bis 2018. Verantwortlichkeiten, Ursachen und Handlungsempfehlungen, 2021, 434, einzusehen unter https://mam.erzbistum-koeln.de/m/2fce82aof87eeo7o/original/ 
soll bewusst hinter eine Vereindeutigung der Darstellung zurückgetreten und stattdessen in Anlehnung an die Methode der "dichten Beschreibung“ herausgearbeitet werden, welche Akteur:innen im Erzbistum Paderborn den Umgang mit Klerikern beeinflussten, die sexuelle Gewalt gegen Minderjährige ausübten. ${ }^{6}$ Ihre Teilhabe, Erfahrungen und Wahrnehmungen werden anhand der Taten einer Einzelperson, des Klerikers Franz Doneiser, geschildert und gedeutet.

Der 1912 in Breslau geborene Franz Doneiser arbeitete nach Abschluss seines Studiums als Seelsorger und Religionslehrer in Schlesien, bevor er 1940 als Soldat eingezogen wurde. ${ }^{7}$ Nach Kriegsende blieb er bis 1946 in sowjetischer Gefangenschaft. Im Anschluss wechselte der Vierunddreißigjährige wie rund 100 weitere schlesische Priester in das Kommissariat Magdeburg. Dieses Gebiet gehörte seit den 182oer Jahren zum (Erz)Bistum Paderborn und wurde durch einen (erz)bischöflichen Kommissar verwaltet. ${ }^{8}$ Zum Einflussbereich des Paderborner Erzbischofs gehörten für den hier relevanten Untersuchungszeitraum von Mitte der 195oer Jahre bis Mitte der 197oer Jahre demnach Gebiete in der Bundesrepublik und in der DDR.

Nach eigener Aussage hatte Doneiser während seinerTätigkeit in der Diözese Breslau keine sexuelle Gewalt gegen Minderjährige ausgeübt. Im Kommissariat Magdeburg war er zunächst als Aushilfe in der Pfarrei Oschersleben in einem Waisenhaus tätig9 ${ }^{9}$ und ab September 1947 als Kaplan in Weißenfels im Bezirk

Gutachten-Pflichtverletzungen-von-Diozesanverantwortlichen-im-Erzbistum-Koln-imUmgang-mit-Fallen-sexuellen-Missbrauchs-zwischen-1975-und-2018.pdf, zuletzt aufgerufen am 4.4.2021; Peter-Andreas Brand/Sabine Wildfeuer, Sexueller Missbrauch an Minderjährigen durch katholische Priester, Diakone und männliche Ordensangehörige im Bereich des Erzbistums Berlin seit 1946. Berlin 2021, einzusehen unter https://www.erzbistumberlin.de/ fileadmin/user_mount/PDF-Dateien/Erzbistum/ErzbischofKoch/20210129GutachtenSexuel lerMissbrauch.pdf, zuletzt aufgerufen am 5.4.2021; Ulrich Wastl/Martin Pusch/Nata Gladstein, Sexueller Missbrauch Minderjähriger und erwachsener Schutzbefohlener durch Kleriker im Bereich des Bistums Aachen im Zeitraum 1965 bis 2019. München 2020, einzusehen unter https://westpfahl-spilker.de/wp-content/uploads/2020/11/Gutachten_Bistum_Aachen.pdf, zuletzt aufgerufen am 20.11.2020.

6 Clifford Geertz, Dichte Beschreibung. Beiträge zum Verstehen kultureller Systeme. Frankfurt a. M. 2003 .

7 Personalbogen, Erzbistumsarchiv Paderborn (EBAP): Personalakte (PA) 414.

8 Hans Jürgen Brandt/Karl Hengst, Geschichte des Erzbistums Paderborn, Bd. 4: Das Bistum Paderborn 1930-2010. Paderborn 2014, 36-38; dies., Geschichte des Erzbistums Paderborn, Bd. 3: Das Bistum Paderborn im Industriezeitalter 1821-1930. Paderborn 1997, 145-147.

9 Karteikarte Anstellungen, EBAP: PA 414; Psychiatrisches Gutachten, 3.8.1967, EBAP: Sonderakte (SA) 124 . 
Halle. ${ }^{10}$ Dort gehörten Unterricht und Jugenderziehung zu seinen Aufgaben. ${ }^{11}$ Sowohl in Weißenfels als auch an seinem zweiten Einsatzort in Sandau übte Doneiser nachweislich sexuelle Gewalt gegen Minderjährige aus und erhielt hierfür zwei Haftstrafen. Ein weiterer sexueller Übergriff ist aus dem Bezirkskrankenhaus Uchtspringe dokumentiert, in das Doneiser nach seiner zweiten Haftstrafe eingewiesen wurde. Das Zusammenspiel von Akteur:innen aus Kirche, Gerichtsbarkeit und Psychiatrie ermöglichte nach diesem Vorfall die Übersiedlung Doneisers in die Bundesrepublik.

Der Fall des Klerikers Doneiser zeigt prägnant, welche Netzwerke das Erzbistum Paderborn in den 196oer und 1970er Jahren zu mobilisierten versuchte, um eine innerkirchliche Auseinandersetzung mit dem Täter zu verhindern. In einer alltagshistorischen Herangehensweise soll beschrieben werden, welche Interessen jene Personen verfolgten, die mit dem Fall befasst waren, und welche situativen Handlungsräume sie besaßen. ${ }^{12} \mathrm{Zu}$ diesem Kreis zählte nicht nur das Leitungspersonal des Paderborner Erzbistums, sondern auch weitere weltliche und geistliche Angehörige des Bistums, ferner Jurist:innen und Psychiater:innen in Ost und West. Ihre Wissenstraditionen und die Stationen Doneisers in der DDR und der Bundesrepublik stehen im Mittelpunkt dieses Beitrags.

Zusätzlich zu Quellen aus dem Erzbistumsarchiv Paderborn (EBAP) werden Dokumente berücksichtigt, die im Zusammenhang der Ermittlungen gegen Doneiser in der DDR entstanden und im Landesarchiv Sachsen-Anhalt (LASA) sowie in der Behörde des Bundesbeauftragten für die Stasiunterlagen (BStU) überliefert sind. Diese Dokumente ergänzen die nur dünne Überlieferung des EBAP zu den Straftaten Doneisers sowie deren innerkirchliche und strafrechtliche Verfolgung. Da die Überlieferung teils in spezifischen Machtkonstellationen (Gerichtsverhandlungen, psychiatrischen Begutachtungen, polizeilicher Beobachtung) entstanden ist, werden im Folgenden der jeweilige Entstehungskontext der Aussagen hervorgehoben und unterschiedliche Perspektiven nebeneinandergestellt. Die Ursachen für die Lücken in den EBAP-Akten lassen sich nicht rekonstruieren. Sicher ist, dass ein Teil der Kommunikation zwischen dem Erzbischöflichen Generalvikariat in Paderborn und dem Erzbischöflichen Kommissariat in Magdeburg mündlich erfolgte. Auf Magdeburger Seite enthalten Personalakten aufgrund der bis 1993

10 Beauftragung Bischöfliches Kommissariat Magdeburg, 13.9.1947, EBAP: SA 124; Karteikarte Anstellungen, PA 414.

11 Lebenslauf, o.D., LASA: M 81 Nr. P 1031, 48-53.

12 Alf Lüdtke, Einleitung. Was ist und wer treibt Alltagsgeschichte?, in: ders. (Hrsg.), Alltagsgeschichte. Zur Rekonstruktion historischer Erfahrungen und Lebensweisen. Frankfurt a. M./New York 1989, 9-47, hier 14. 
praktizierten Kassationspraxis nur basale Angaben..$^{13} \mathrm{Ob}$ im EBAP einzelne Dokumente aus den Akten entfernt wurden, kann aufgrund fehlender Paginierung nicht entschieden werden.

\section{Taten in Weißenfels, Bezirk Halle (195oer Jahre)}

Seit dem Ende der nationalsozialistischen Herrschaft und seiner Rückkehr aus der Gefangenschaft fühlte sich Franz Doneiser nach eigener Aussage von minderjährigen Jungen sexuell angezogen. ${ }^{14}$ Zunächst habe er sich selbst befriedigt, dann jedoch sexuelle Enthaltsamkeit und den Verzicht von Nikotin und Alkohol angestrebt, also entsprechend zeitgenössischer Normen Selbstbeherrschung geübt. Gegenüber seiner vorgesetzten Behörde sprach er seine Probleme nur in allgemeiner Form an, indem er über psychische und physische Erschöpfung klagte, fand hierfür aber keine offenen Ohren..$^{15}$ Die überlieferten Akten legen nahe, dass Doneiser keine Hilfe suchte, seine sexuellen Fantasien zu bekämpfen. Diese Beobachtung lässt auf die Sagbarkeitsgrenzen schließen, die in dieser Zeit existierten und es potenziellen Tätern erschwerten, im Vorfeld um Hilfe zu ersuchen.

Als Vikar in Weißenfels baute Doneiser gezielt Vertrauen zu Kindern und ihren Eltern auf, indem er die Rolle eines pädagogischen Beraters einnahm. Es ist davon auszugehen, dass Doneiser durch sein Amt als Kleriker ein hohes Ansehen in den Familien der betroffenen Kinder genoss. Dass berufliche und persönliche Kontakte bei sexuellem Missbrauch eng miteinander verwoben sind, ist für sexuelle Gewalt im institutionellen Kontext nicht untypisch. ${ }^{16}$ Einigen Kindern wandte er sich offenbar in besonderer Weise zu. So lud Doneiser mindestens einen Jungen in seine Wohnung ein unter dem Vorwand, ihm bei den Hausaufgaben zu helfen. ${ }^{17}$ Erst nachdem er das Vertrauen des Kindes besaß, begann Doneiser mit sexuellen Gewalthandlungen. Er fasste an das Geschlechtsteil des Jungen und nötigte den Jungen zu solchen

\footnotetext{
13 Schriftliche Auskunft des Bistumsarchiv Magdeburg (BAMa) vom 11.1.2021.

14 Lebenslauf, o.D., LASA: M 81 Nr. P 1031, 48-53.

15 Ebd.

16 Gerhard Hackenschmied/Peter Mosser, Untersuchung von Fällen sexualisierter Gewalt im Verantwortungsbereich des Bistums Hildesheim - Fallverläufe, Verantwortlichkeiten, Empfehlungen, 2017, 74, einzusehen unter https:/www.bistum-hildesheim.de/fileadmin/ dateien/PDFs/Pressetexte/IPP_Muenchen_Gutachten_Bistum_Hildesheim.pdf, zuletzt aufgerufen am 3.1.2021.

17 Lebenslauf, o.D., LASA: M 81 Nr. P 1031, 48-53.
} 
Handlungen bei sich. ${ }^{18}$ Der Minderjährige versuchte, sich den Übergriffen zu entziehen, so habe er, wie Doneiser in seiner psychiatrischen Begutachtung 1967 angab, "gelegentlich [...] protestiert, weil ich doch Kaplan sei. ${ }^{“ 19}$ Die Wahrnehmung des Jungen unterstreicht die strukturell verankerte Macht von Klerikern gegenüber Laien. Das ,Amtscharisma' förderte bei den Betroffenen „konsequenten Gehorsam, auch gegen eigene Interessen.“20 Darin liegt ein spezifisches Moment sexueller Gewaltausübung von Geistlichen. Trotz der Abwehr des Jungen setzte Doneiser seine sexuellen Übergriffe fort.

Dieser Junge war nicht der einzige Minderjährige, gegen den Doneiser sexuelle Gewalt ausübte. Über die anderen Betroffenen geben die vorhandenen Akten nur wenig Auskunft. Über drei bis vier Jahre hinweg missbrauchte Doneiser mehrere Jungen und ein Mädchen im Alter von neun bis 16 Jahren, die er als Religionslehrer und Jugendleiter betreute. Mit den Jungen habe er, so das psychiatrische Gutachten von 1965 , ,vorwiegend gegenseitige Onanie betrieben, auch an einem Mädchen hatte sich D. unsittlich vergangen."21 Diese Aussage aus dem Gutachten ist nur eines von vielen Beispielen, wie die Machtund Gewaltverhältnisse in der Sprache zuweilen beschönigt werden, wenn z.B. von gegenseitiger Onanie die Rede ist. Damit werden die Betroffenen zu sexuell begierigen Subjekten gemacht, die ihre sexuelle Lust befriedigen. Zwei Jahre später, 1967, erweiterte Doneiser im Rahmen der zweiten psychiatrischen Begutachtung in der Bundesrepublik seine Ausführungen zu den Opfern. Er gab an, zwischen $195^{2}$ und 1954 an jedem Ort, an dem er tätig war, gegenüber drei bis vier Jungen sexuell übergriffig gewesen zu sein. Dies deutet darauf hin, dass vor Gericht weder die Gesamtzahl der Betroffenen noch die Intensität der Taten bekannt wurden.

Für die ihm nachgewiesenen Taten wurde Doneiser 1954 wegen sexuellen Missbrauchs von Kindern im besonders schweren Fall ${ }^{22}$, schwerer Unzucht

18 Ebd.

19 Psychiatrisches Gutachten, Landeskrankenhaus (LKH) Marsberg, 3.8.1967, EBAP: SA 124.

20 Gregor Maria Hoff, Kirche zu, Problem tot! Theologische Reflexionen zum Missbrauchsproblem in der katholischen Kirche, in: Religion zum Teufel. Kursbuch 196, 2018, 26-41, hier 34 .

21 Nervenfachärztliches Gutachten, Bezirkskrankenhaus (BK) Uchtspringe, 3.9.1965, LASA: M 81 Nr. P 1031, 8-15. Hervorhebung durch die Autorin.

$22 \S \S 176$ Abs 1 Ziffer 3 StGB. Siehe zum StGB in der DDR Benjamin Baumgart, Juristische Hintergründe zum sexuellen Missbrauch in der DDR, in: Christian Sachse/Stefanie Knorr/Benjamin Baumgart, Sexueller Missbrauch in der DDR. Historische, rechtliche und psychologische Hintergründe des sexuellen Missbrauchs an Kindern und Jugendlichen in der DDR. Wiesbaden 2018, 133-172, hier 135 . 
zwischen Männern ${ }^{23}$ und sexuellen Missbrauchs von Schutzbefohlenen ${ }^{24}$ zu acht Jahren Zuchthaus verurteilt. ${ }^{25}$ Wie bei Strafverfahren gegen Kleriker üblich, beobachtete ein Geistlicher den Prozess im Auftrag des Erzbistums. Dieser berichtete: „Die Verhandlung war hart, aber sachlich. Der Staatsanwalt stellte sehr klar heraus, dass man zwischen Katholischer Kirche und einem Angehörigen derselben unterscheiden müsse.“26

\section{Fehlende Konsequenzen des Erzbistums}

Aufgrund einer DDR-weiten Amnestie wurde Doneiser am 23. Dezember 1960 vorzeitig aus der Haft entlassen. Der Paderborner Erzbischof Lorenz Jaeger $(1892-1975)^{27}$ erfuhr von der Begnadigung und richtete an Doneiser eine Woche nach der Haftentlassung einen Brief: „Mit Ihnen danke ich Gott dem Herrn, daß nunmehr die schwere Zeit Ihrer Haft beendet ist und ein neuer Abschnitt Ihres Lebens beginnen kann. Lassen Sie nun alles hinter sich, was gewesen ist! Schauen Sie nur nach vorn, zu der Aufgabe hin, zu der der Herr sie ruft! ${ }^{\prime 28}$ Die im Erzbistumsarchiv Paderborn überlieferten Dokumente deuten darauf hin, dass eine kirchengerichtliche Untersuchung und Sanktionierung der Taten unterblieben. Vielmehr sollte Doneiser nach seiner „Prüfungszeit“29, gemeint ist die Haft, als Hausgeistlicher ein neuer Anfang ermöglicht werden.

Im Februar 1961 betraute Friedrich Maria Rintelen (1899-1988), der Erzbischöfliche Kommissar in Magdeburg ${ }^{30}$, Doneiser mit der Seelsorge in einem Altersheim in Sandau. Vor Stellenantritt lud Rintelen ihn nach Magdeburg ein. ${ }^{31}$ Der Inhalt dieses Gesprächs ist in den Akten des Erzbistums nicht dokumentiert. Aus einem späteren Brief Rintelens geht hervor, dass Doneiser

$23 \S 175$ a Ziffer 2 StGB.

$24 § 174$ StGB.

25 Strafregistrierkartei Franz Doneiser, BStU: AR2.

26 Pfarrer Holzem an Jaeger, 11.5.1954, EBAP: SA 124.

27 Zu Lorenz Kardinal Jaeger wird aktuell im Auftrag der Kommission für Kirchliche Zeitgeschichte im Erzbistum Paderborn und unter Leitung von Prof. Dr. Nicole Priesching ein Forschungsprojekt durchgeführt. Siehe hierzu https://kw.uni-paderborn.de/institutfuer-katholische-theologie/kirchen-und-religionsgeschichte/forschungsprojekte/ projekte-des-lehrstuhls/aufarbeitung-des-nachlasses-von-lorenz-kardinal-jaeger, zuletzt aufgerufen am 20.05.2021.

28 Jaeger an Doneiser, 1.12.196o, EBAP: SA 124.

29 Schreiben Piontek [Bischof und Kapitelsvikar Görlitz], 26.6.1961, EBAP: PA.

30 Siehe zu Rintelen: Brandt/Hengst, Bistum: Bd. 4, $136 f$.

31 Rintelen an Doneiser, 14.2.1961, EBAP: SA. 
die Seelsorge auf das Altenheim beschränken sollte. ${ }^{32}$ In dem 1965 erstellten psychiatrischen Gutachten gab ein Geistlicher der Gemeinde Sandau an, dass Doneiser verboten wurde, „Jugendstunden durchzuführen und in Familien, die Kinder haben, zu verkehren. ${ }^{33}$ Dies legt nahe, dass disziplinarische Maßnahmen ergriffen wurden. Allerdings sei, so der Sandauer Geistliche weiter, der "Lebenswandel“ Doneisers nicht immer "gut genug zu kontrollieren“34 gewesen und Doneiser habe "gegen das ausgesprochene Verbot mit der Familie des geschädigten Knaben freundschaftlichen Umgang gepflegt.“35

Dieser Darstellung zufolge hatte sich Doneiser den Vorgaben der vorgesetzten Behörde entzogen. Sie unterschlägt jedoch, dass das Erzbischöfliche Kommissariat in Magdeburg durchaus Kenntnis von Doneisers seelsorgerischen Tätigkeit außerhalb des Altenheims gehabt haben konnte. Nach einem Bericht der Volkspolizei habe dieser "die in Sandau lebenden Katholiken“ ${ }^{36}$ betreut. Ferner half er, so das Gutachten aus dem Jahr 1965, vertretungsweise „bei Sonntagsgottesdiensten in näherer und weiterer Umgebung aus.“37 Dies wäre ohne Kenntnis des Pfarrers von Klietz, zu dessen Gebiet Sandau gehörte und der in den Außenbezirken regelmäßige Gottesdienste abzuhalten hatte, nicht möglich gewesen. Die Außenstellen der Pfarrei Klietz lagen weit auseinander und der Pfarrer besaß lediglich die Unterstützung eines Vikars. Bis nach Sandau waren es von Klietz 14 Kilometer, der weiteste Weg führte in das 28 Kilometer entfernte Schollene. ${ }^{38}$ Die Übernahme von Gottesdiensten durch Doneiser erleichterte demnach die Arbeit des Klietzer Pfarrers. Ob er Kenntnis von Doneisers Vorstrafe hatte, ist nicht bekannt. Dennoch kann festgehalten werden, dass eine Aufsicht durch den örtlichen Pfarrer vom Erzbischöflichen Kommissariat nicht angeordnet oder nicht durchgeführt wurde.

Wenngleich die hier zitierten Dokumente im Kontext der zweiten Gerichtsverhandlung entstanden und im weitesten Sinne als „Herrschaftswissen“ über den Bürger und psychiatrischen Patienten Doneiser aufzufassen sind, so zeigt die Zusammenschau doch ein hohes Maß an Übereinstimmung: Doneiser sollte, obgleich er jahrelang sexuelle Gewalt gegen Minderjährige ausgeübt hatte, eine neue Chance erhalten. Als Maßnahme zum Schutz weiterer Kinder erteilte das Erzbischöfliche Kommissariatlediglich mündlich Umgangsverbote,

\footnotetext{
32 Bericht Rintelen an Generalvikariat, 1.6.1965, EBAP: SA 124.

33 Nervenfachärztliches Gutachten, BK Uchtspringe, 3.9.1965, LASA: M 81 Nr. P 1031, 8-15.

34 Ebd.

35 Ebd.

36 Bericht Volkspolizei Sandau, 29.5.1964, BStU: MfS B-SKS 36064.

37 Nervenfachärztliches Gutachten, BK Uchtspringe, 3.9.1965, LASA: M 81 Nr. P 1031, 8-15.

38 Visitationsbogen Klietz (1962), BAMa: I - 56/1: Visitationsberichte des Dechanten. Dekanat Stendal.
} 
überwachte diese jedoch nicht. Dadurch erhielt Doneiser in seinem neuen Wirkungsfeld in Sandau ein Höchstmaß an Handlungsfreiheit.

\section{Taten in Sandau, Bezirk Stendal (196oer Jahre)}

Doneiser lernte die Familie seines späteren Opfers nach rund zweijähriger Tätigkeit in Sandau im Sommer 1963 in jenem Pflegeheim kennen, in dem er als Seelsorger wirkte. ${ }^{39}$ In seinem Lebenslauf gab Doneiser an, dass er die Familie „fast monatlich“40 besuchte. Erneut nahm er, wie zuvor in Weißenfels, die Rolle eines Ratgebers der Familie ein. Er unterstützte sie durch Geschenke und erteilte dem späteren Opfer Nachhilfeunterricht. Mit seinen eigenen Worten ausgedrückt, litt Doneiser ab 1964 unter „sexuelle[n] Spannungen“.41 Zwar berichtete er im Rahmen der psychiatrischen Begutachtung, dass er zunächst erfolglos versucht habe, Begegnungen zu dem Jungen zu vermeiden, suchte aber offenbar keine weitere Hilfe. Doneiser nährte sich dem Jungen schrittweise an, unternahm gemeinsame Spaziergänge, unterhielt sich, spielte mit ihm und versuchte, ihn durch kleinere Geschenke an sich zu binden. Sexuelle Übergriffe erfolgten zunächst durch vermeintlich spielerische Handlungen und durch, wie es die Staatsanwaltschaft Havelberg unter Negierung der Machtverhältnisse formulierte, „erotische Gespräche “42, die bereits die Intimsphäre des Jungen verletzten. Die Anbahnung und Durchführung der von Doneiser mehrfach und über einen langen Zeitraum ausgeübten sexuellen Gewalt folgte einer typischen Täterstrategie, nämlich spätere Opfer unter denjenigen zu suchen, die sich mit Problemen vertrauensvoll an Funktionsträger gewandt hatten. ${ }^{43}$ Die daraus resultierenden emotionalen Bindungen erschwerten es vielen Betroffenen, sich aus dem Abhängigkeitsverhältnis zu lösen. ${ }^{44}$

39 Urteil Kreisgericht Havelberg, S $36 / 65$ K I B 50/65, LASA: M 81 Nr. P 1031, 7off; Lebenslauf, o.D., LASA: M 81 Nr. P 1031, 48-53.

40 Lebenslauf, o.D., LASA: M 81 Nr. P 1031, 48-53.

41 Ebd.

42 Anklageschrift, 9.6.1955, BStU: MfS B-SKS 36064; Lebenslauf, o.D., LASA: M 81 Nr. P 1031, 48-53; Urteil Kreisgericht Havelberg, S $36 / 65$ K I B 5o/65, LASA: M 81 Nr. P 1031, 7 off.

43 Siehe beispielhaft: Bistum Limburg (Hrsg.), Projektdokumentation. Betroffene hören Missbrauch verhindern. Konsequenzen aus der MHG-Studie, 2020, 170f., einzusehen unter https://bistumlimburg.de/fileadmin/redaktion/Portal/Meldungen/2020/202O_o6_13_ Abschluss_MHG/Dateien_zum_Download/2020-o6-17_Abschlussbericht_online.pdf, zuletzt eingesehen am 20.11.2020.

44 Norbert Leygraf u.a., Sexuelle Übergriffe durch katholische Geistliche in Deutschland. Eine Analyse forensischer Gutachten 2000-2020. Abschlussbericht 2012, 30, einzusehen unter https://www.dbk.de/fileadmin/redaktion/diverse_downloads/Dossiers_ 
Offenbar gelang dies dem betroffenen Jungen, denn er konnte dem Gericht zahlreiche sexuelle Übergriffe schildern. Dennoch erkannte das Gericht nicht alle Angaben des Opfers als erwiesen an. Unter anderem hielt das Gericht eine Tat vom September 1964 für glaubhaft. An diesem Tag entblößte sich Doneiser vor dem Jungen und onanierte bis zur Ejakulation. ${ }^{45}$ Das Gericht war zwar der Auffassung, dass Doneiser in der Folge versucht habe, weitere Missbrauchshandlungen zu begehen, folgte aber entgegen der Darstellung des Kindes, dass solche Übergriffe „häufiger ${ }^{446}$ stattfanden, der Schilderung des Angeklagten, bzw. seines Verteidigers, dabei immer gestört worden zu sein. In der Folge sei es zu Berührungen des Geschlechtsteils oberhalb der Kleidung gekommen. Zu einer weiteren aus Sicht des Gerichtes nachweisbaren Handlung kam es am 18. Mai 1965, als Doneiser erneut vor dem Jungen masturbierte. ${ }^{47}$ Laut juristischer Abklärung hatte Doneiser zudem Druck auf den Jungen ausgeübt. Denn er forderte sein Opfer auf, über die Taten zu schweigen und gab ihm dafür Geld sowie Süßigkeiten. ${ }^{48}$ Als Doneiser zwei Jahre später in der Bundesrepublik psychiatrisch begutachtet wurde, berichtete er, den Jungen über ein halbes Jahr hinweg ca. einmal die Woche zu sexuellen Handlungen, darunter zu Oralverkehr, gezwungen zu haben. Dabei habe es sich um zehn bis fünfzehn Taten gehandelt. ${ }^{49}$ Angesichts der fast wöchentlich erfolgten sexuellen Gewalthandlungen über sechs Monate hinweg erscheint diese Angabe als zu niedrig. Aus diesen Hinweisen folgt, dass wiederum nur ein Teil der Taten strafrechtlich geahndet wurde.

Schließlich wurde Doneiser wegen sexuellen Missbrauchs von Kindern im besonders schweren Fall ${ }^{50}$ zu einer Haftstrafe von einem Jahr verurteilt ${ }^{51}$ und, im Unterschied zum ersten Prozess, eine anschließende Sicherungsverwahrung angeordnet. Das Gericht ging auf Grundlage des psychiatrischen Gutachtens davon aus, dass Doneiser rückfallgefährdet sei. ${ }^{52}$ Daher sei es im „Interesse des Schutzes der Kinder und der öffentlichen Sicherheit [...] erforderlich, die Behandlung in einer Heil- und Pflegeanstalt durchzuführen. “53

2012/2012_Sex-Uebergriffe-durch-katholische-Geistliche_Leygraf-Studie.pdf, zuletzt aufgerufen am 20.12.2O2O.

45 Urteil Kreisgericht Havelberg, S $36 / 65$ K I B 5o/65, LASA: M 81 Nr. P 1031, 7off.

46 Ebd.

47 Ebd.

48 Ebd.

49 Psychiatrisches Gutachten, LKH Marsberg, 3.8.1967, EBAP: SA 124.

50 $§ 176$ Abs. 1, Ziffer 3 StGB.

51 Urteil Kreisgericht Havelberg, S $36 / 65$ K I B 50/65, LASA: M 81 Nr. P 1031, 7off.

$5^{2}$ Das Archiv stellte der Autorin Exzerpte des Gutachtens bezüglich der Straftaten Doneisers zur Verfügung.

Urteil Kreisgericht Havelberg, S 36/65 K I B 5o/65, LASA: M 81 Nr. P 1031, 7off. 
Damit wurde neben dem Strafgesetzbuch auch das psychiatrische Gutachten Grundlage der juristischen Verurteilung Doneisers.

Der Philosoph Michel Foucault sah eine Funktion von psychiatrischen Gutachten darin, dass sie, anders als das Gericht, einen Zusammenhang zwischen Tat und Wesen konstituieren können. ${ }^{54}$ Diesem Unterschied liegt eine Entwicklung zugrunde, die im Wesentlichen von Psychiatern des 19. Jahrhunderts getragen wurde. Der Psychiater Bénédict Augustin Morel (1809-1873), der in seiner Jugend ein Priesterseminar besucht hatte, deutete abweichende Lebensstile, darunter sexuelle Praktiken, die nicht der Fortpflanzung dienten, unterschiedslos als Ausdruck moralischer Degeneration. Wenngleich Morel, anders als spätere Vertreter der Degenerationslehre, zusätzlich zur Vererbbarkeit von solchen „Degenerationsmerkmalen“ ebenso Umwelteinflüsse als ursächlich betrachtete, legte er doch den Grundstein eines psychiatrischen Denkens, das Vererbung als „Wesensmerkmal beinahe aller Geisteskrankheiten“ ${ }^{45}$ ansah. Abweichendes Verhalten, für das die Kirche Buße auferlegen und Vergebung gewähren konnte, deuteten zahlreiche Psychiater:innen bis in den hier relevanten Zeitraum hinein als Ausdruck unheilbarer Krankheiten, beispielsweise mit der Diagnose Psychopathie. Die körperliche Konstitution, die Arbeitsfähigkeit und das Sexualverhalten, das nicht der ,Fortpflanzung‘ diente, avancierten zu zentralen Kategorien bei der gerichtspsychiatrischen Bewertung der Zurechnungsfähigkeit. ${ }^{6}$ Erst diese Entwicklung erklärt eine dreifache Perspektive auf Franz Doneiser als Straftäter, Kranker und Sünder.

\section{Grenzüberschreitende Handlungskooperation im Feld von Kirche, Justiz und Psychiatrie}

Am 25. Mai 1966 erfolgte nach Verbüßen der Haftzeit die Überstellung Doneisers in das Bezirkskrankenhaus Uchtspringe. ${ }^{57} \mathrm{Im}$ Oktober desselben Jahres wurde er bei sexuellen Handlungen mit einem anderen Patienten in einem nahegelegenen Wald erwischt und „[a]us Sicherheitsgründen“58 auf eine geschlossene Station verlegt. Anhand der Überlieferung lässt sich über

\footnotetext{
54 Michel Foucault, Die Anormalen. 4. Aufl. Frankfurt a. M. 2016, 33.

55 Anna Bergmann, Genealogien von Gewaltstrukturen in Kinderheimen, in: Michaela Ralser/Reinhard Sieder (Hrsg.), Die Kinder des Staates. (Österreichische Zeitschrift für Geschichtswissenschaften 25.) Innsbruck 2014, 82-116, hier 97. Siehe auch ebd., 95ff.

56 Anna Bergmann, Die verhütete Sexualität. Die Anfänge der modernen Geburtenkontrolle. Hamburg 1992, 140.

57 BK Uchtspringe an Bezirksbehörde der Volkspolizei, 12.2.1966, LASA: M 81 Nr. P 1031, 67.

58 Vermerk in Krankenakte vom 11.10.1966, LASA: M 81 Nr. P 1031. Auskunft durch das Archiv.
} 
das Alter des Mitpatienten keine Aussage treffen. Generalvikar Joseph Droste (1911-1991) 59 schrieb von einem "Jungen“, mit dem Doneiser „in flagranti“ 60 erwischt wurde, der Erzbischöfliche Kommissar Rintelen von einem "Jung-

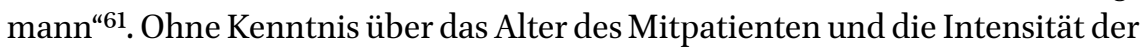
sexuellen Handlungen, kann nicht entschieden werden, ob diese nach DDRRecht justiziabel waren. ${ }^{62}$ Die differierenden Altersangaben unterstreichen zudem die fehlenden Bemühungen des Erzbistums, den Sachverhalt näher zu untersuchen.

Trotz oder möglicherweise auch gerade wegen dieses erneuten Vorfalls sind seit November 1966 Versuche dokumentiert, die Entlassung Doneisers aus der Klinik in die Wege zu leiten. Zu den Akteuren gehörten leitende Angehörige des Erzbistums: auf Paderborner Seite Erzbischof Lorenz Jaeger und der Generalvikar Joseph Droste sowie auf Magdeburger Seite der Erzbischöfliche Kommissar Rintelen. Die folgende Analyse des Briefverkehrs lenkt den Blick darüber hinaus auch auf weitere einflussnehmende Akteur:innen.

Wo die Initiative zur Entlassung Doneisers aus der Klinik ihren Anfang nahm, kann nicht genau rekonstruiert werden. Das erste überlieferte schriftliche Dokument in diesem Zusammenhang ist ein Schreiben des Bezirkskrankenhauses Uchtspringe vom 2. November 1966 an das Bischöfliche Ordinariat Magdeburg. Die Klinik strebte an, bei der zuständigen Staatsanwaltschaft „einen Antrag auf bedingte Aussetzung der Sicherungsverwahrung $^{\text {“63 }}$ zu stellen, wenn eine kirchliche Einrichtung benannt werden könne, „die eine ausreichende Beaufsichtigung dieses durch seine sexuellen Perversionen weiterhin relativ gefährdeten ehemaligen Geistlichen gewährleisten" kann. ${ }^{64}$ Solche Absprachen wurden auch in anderen Fällen, sowohl im Erzbistum Paderborn als auch in anderen Diözesen, getroffen. ${ }^{65}$ Am selben Tag, als der Brief aus dem Krankenhaus an Rintelen verfasst wurde, informierte dieser seinerseits das Generalvikariat Paderborn über die sexuellen Handlungen Doneisers. ${ }^{66}$ Die weitere Abstimmung zwischen Rintelen und dem Generalvikariat erfolgte offenbar mündlich. Denn schon am

\footnotetext{
59 Siehe zu Droste: Brandt/Hengst, Erzbistum: Bd. 4, 122.

6o Droste an Jahn, 17.6.1967, EBAP: SA 124.

61 Rintelen an Generalvikariat, 20.2.1967, EBAP: SA 124.

62 Siehe zur Rechtslage und -auslegung in der DDR der 196oer Jahre Alexander Zinn, ,Gegen das Sittengesetz: Staatliche Homosexuellenverfolgung in Deutschland 1933-1969, in: ders. (Hrsg.), Homosexuelle in Deutschland 1933-1969. Göttingen 2020, 15-47, hier 4of.

63 BK Uchtspringe an das Bischöfliche Ordinariat Magdeburg, 2.11.1966. Eine Abschrift ging an das Erzbistum Paderborn. EBAP: SA 124.

64 Ebd. Doneiser war zu diesem Zeitpunkt keineswegs laisiert.

65 EBAP: SA 93; Gercke u.a., Gutachten, 434.

66 Rintelen an Droste, 2.11.1966, EBAP SA 124.
} 
4. November teilte Rintelen dem Bezirkskrankenhaus Uchtspringe mit, dass es keine entsprechende Unterbringungsmöglichkeit im Kommissariat gäbe, der Erzbischof aber bereit sei, Doneiser „im Westteil des Erzbistums in einer geschlossenen Anstalt so unterzubringen, daß nach menschlichem Ermessen [...] keine Möglichkeit zu neuen Straftaten gegeben sein würde.“" E7 Erzbischof Jaeger stellte jedoch zwei Bedingungen: Der westdeutschen Staatsanwaltschaft sollten keine Akten zugehen und Doneiser sollte eine legale Ausreise ermöglicht werden. Das Erzbistum wollte auf diese Weise vermeiden, wie es in dem Schreiben hieß, „daß durch die Straftaten des ehemaligen Vikars auch in Westdeutschland ein Skandalum entsteht.“68 Zusätzlich übte das Erzbistum Druck auf Doneiser aus, einen Antrag auf Laisierung zu stellen. ${ }^{69}$ Ungeachtet dessen, dass die Aushändigung von Akten bei der Ausreise von straffällig gewordenen DDR-Bürger:innen als unwahrscheinlich anzusehen ist, stehen die von Jaeger formulierten Bedingungen einzig unter der Prämisse, das Bekanntwerden der Straftaten Doneisers und dessen Zugehörigkeit zum Erzbistum zu verschleiern.

Angesichts der erzbischöflichen Zusage stellte das Bezirkskrankenhaus Uchtspringe am 9. November 1966 bei der zuständigen Staatsanwaltschaft einen Antrag auf Aussetzung der Sicherungsverwahrung. Damit einhergehend wurde das politische Interesse formuliert, dass so „die auch für uns optimale Lösung einer Übersiedlung nach Westdeutschland dieses kriminell gewordenen Klerikers ermöglicht werden kann." ${ }^{\text {"70 }}$ Ohne weitere Quellen ist nicht zu entscheiden, ob neben den vorgebrachten politischen Erwägungen ebenso pragmatische Interessen für die vorgeschlagene Entlassung eine Rolle gespielt haben, da die psychiatrischen Kliniken der DDR in dieser Zeit unter Überbelegung, Personalmangel und baulichen Mängeln litten. Dies galt ebenso für die Bundesrepublik. ${ }^{71}$ Der Staatsanwalt des Kreises leitete die Anfrage an den Bezirksstaatsanwalt weiter. Dieser lehnte den Antrag am 8. Dezember 1966 ab, „da eine Besserung des Gesundheitszustandes des D.[oneiser] nicht eingetreten [ist], so daß nach wie vor damit zu rechnen ist, daß D.[oneiser] erneut rückfällig wird." ${ }^{\text {"72 }}$

Eine gute Woche später wiederholte das Bezirkskrankenhaus Uchtspringe den Antrag und konstatierte nun trotz des erst vier Monate zurückliegenden

\footnotetext{
67 Rintelen an BK Uchtspringe, 4.11.1966, EBAP SA 124.

68 Ebd.

69 Droste an Rintelen, 28.2.1967, EBAP: SA 124.

$70 \quad$ BK Uchtspringe an Staatsanwaltschaft Havelberg, 9.11.1966. LASA: M 81 Nr. P 1031, 20.

71 Sabine Hanrath, Zwischen ,Euthanasie und Psychiatriereform: Anstaltspsychiatrie in Westfalen und Brandenburg. Ein deutsch-deutscher Vergleich (1945-1964). Paderborn u.a. $2002,275^{-285}$ und $369-400$.

72 Staatsanwalt Kreis Havelberg an BK Uchtspringe, 8.12.1966, LASA: M 81 Nr. P 1031, 22.
} 
sexuellen Übergriffs „eine leichte Besserung“.73 Da nur wenige zeitgenössische Psychiater:innen überhaupt eine Heilungsaussicht bei Sexualstraftätern annahmen, erscheint es naheliegend, dass diese Feststellung strategischer Natur war. Erneut wurde in das Schreiben der Klinik an die Staatsanwaltschaft ein politisches Argument eingepflegt. Um eine Entlassung Doneisers aus der Klinik zu erreichen, hob der behandelnde Arzt die fehlenden Eingliederungsmöglichkeiten Doneisers in die sozialistische Arbeitsgesellschaft hervor. Damit negierte er zugleich den zuvor konstatierten Heilungserfolg: „Wir hoffen, dass diese Lösung im Falle des Vikar Doneiser Ihre Zustimmung finden wird, was eigentlich der Fall sein müsste, da dieser sonst als langfristig oder gar dauernd sicherungsverwahrter Insasse einer psychiatrischen Einrichtung der DDR und als ein für den Produktionsprozess nicht infrage kommender Bürger nur eine ökonomische Belastung bedeutet. ${ }^{\text {“74 }}$ Die Staatsanwaltschaft, die zunächst keinen Handlungsdruck gesehen hatte, stimmte nun, Anfang Februar 1967, Verhandlungen zwischen der Verwaltung des Bezirks Magdeburg und der „kath. Kirche ${ }^{\text {“75 }} \mathrm{zu}$.

Zeitgleich beriet das Leitungspersonal im Generalvikariat mit dem Erzbischof in Paderborn darüber, wie mit Doneiser nach seiner Ausreise aus der DDR umzugehen sei.ImWesten würde er nach dervomErzbistum angestrebten Laisierung als unbescholtener Bürger gelten. Zudem hatte Doneiser im Gegenzug für die Laisierung die Zusage erhalten, in der Bundesrepublik „möglichst frei leben und arbeiten [...] [zu können], ohne um seine Existenzmöglichkeiten fürchten zu müssen. ${ }^{46}$ Ungeachtet der den DDR-Behörden gegebenen Zusage, Doneiser in einer geschlossenen Anstalt unterzubringen, wurde angestrebt, ihm beispielsweise als Pförtner in einer psychiatrischen Einrichtung Beschäftigung und Unterkunft zu besorgen und so nach außen hin die Beziehung zu kappen. Hierfür reichte der erzbischöfliche Einfluss allerdings nicht aus. Am 1. März 1967 schilderte Generalvikar Droste in einem Schreiben an vier Pfarrer in psychiatrischen Einrichtungen und an einen leitenden Vertreter der Diözesan-Caritas beschönigend, dass im Kommissariat Magdeburg „ein heimatvertriebener Geistlicher [...] nach mehreren Sittlichkeitsdelikten mit Jugendlichen (Homosexualität) und Verbüßung von 2 Freiheitsstrafen unter sehr unangenehmen Verhältnissen in Sicherungsverwahrung

73 BK Uchtspringe an Staatsanwaltschaft des Kreises Havelberg und des Bezirks Magdeburg, 17.12.1967, LASA: M 81 Nr. P 1031, 23.

74 Ebd.

75 Staatsanwaltschaft Bezirk Magdeburg an BK Uchtspringe, 8.2.1967, LASA: M $81 \mathrm{Nr}$. P 1031, 28.

76 Droste an Rintelen, 28.2.1967, EBAP: SA 124. 
gehalten“77 werde. Droste bat um Hilfe für den „unglücklichen Mitbruder“. ${ }^{48}$ Das Schreiben wurde jeweils abschlägig beantwortet. Die Verantwortung, die Straftaten zu verschweigen, wollte keiner der Angeschriebenen übernehmen. Zwei Klinikpfarrer hielten Rücksprache mit dem ärztlichen Leiter ihrer Einrichtungen. Einer riet, zuerst ein psychiatrisches Gutachten anzufertigen und die ,juristischen Voraussetzungen “79 zu klären. Der Pfarrer des Johannesstift in Niedermarsberg betrachtete angesichts der dort untergebrachten Kinder und Jugendlichen die Anstellung Doneisers als „Gefahr ${ }^{480}$, weshalb von anderer Seite die Aufnahme in einem "caritativ, konfessionell geleiteten Heim “81 angeraten wurde. Einen weiteren Aspekt hob der Direktor der DiözesanCaritas hervor, der fürchtete, dass bei einer öffentlich sichtbaren Tätigkeit als Pförtner "schon bald ein Raten und Befragen über die Herkunft einsetzen würde.. 82

Als Ende März 1967 seitens der Magdeburger Staatsanwaltschaft Doneisers Ausreise nichts mehr im Wege stand, war dessen weiterer Aufenthalt in der Bundesrepublik also weiterhin unbestimmt. ${ }^{83}$ Dennoch erklärte das Erzbischöfliche Kommissariat in Magdeburg am 17. April anlässlich der Übersendung von Doneisers Ausreiseantrag: „Das Erzbischöfliche Generalvikariat in Paderborn hat sich [...] schriftlich bereit erklärt, Herrn D[oneiser] in einem entsprechenden Hause unterzubringen, wo er unter Aufsicht steht und eine entsprechende Beschäftigung erhält." ${ }^{84}$ Am 27. Mai 1967 reiste der fünfundfünfzigjährige Doneiser, nachdem er ein von Rintelen aufgesetztes Schreiben mit dem Antrag auf Laisierung unterschrieben hatte, ${ }^{85}$, unbelastet ${ }^{\star}$ in die Bundesrepublik ein: „Herr Doneiser ist geisteskrank und bedarf der ständigen Pflege und Beaufsichtigung und wird deshalb unter Aufsicht des Generalvikariats in Paderborn genommen." ${ }^{\text {"6 }}$ Als Invalide unterstand er nicht dem Ausreiseverbot der DDR. Das psychiatrische Argument der

\footnotetext{
77 Rundschreiben Droste, 1.3.1967, EBAP: SA 124.

78 Ebd.

79 Schreiben Klinikpfarrer Eickelborn, 28.2.1967, EBAP: SA 124.

8o Schreiben Klinikpfarrer St. Johannesstift, 2.3.1967, EBAP: SA 124.

81 Schreiben katholisches Pfarramt Warstein/Sauerland, West. Landeskrankenhaus, 8.3.1967, EBAP: SA 124.

82 Schreiben Diözesan-Caritasdirektor, 16.3.1967, EBAP: SA 124.

83 Jäger an Droste, 7.4.1968, EBAP: SA 124.

84 Erzbischöfliches Kommissariat an Rat des Bezirks Magdeburg, 17.4.1967, LASA: M 1 Nr. 11612, 47.

85 Doneiser an Generalvikariat, 6.4.1967, EBAP: SA 124.

86 Stellvertretender Vorsitzender für Inneres an Bezirksbehörde der Volkspolizei, 20.4.1967, LASA: M 1, Nr. 11612, 46.
} 
Geisteskrankheit des Sexualstraftäters ermöglichte also die Ausreise Doneisers aus der DDR.

Die dargestellten Kommunikationswege umreißen jene Akteure, die in die Übersiedlung Doneisers in die Bundesrepublik involviert waren und welche Interessen sie verfolgten. Im Westteil wurden vor allem Angehörige des Bistums eingeweiht, ohne dass sich der Kreis auf die Leitungsebene beschränkte. Durch Klinikpfarrer und ein Leitungsmitglied der Caritas versuchte das Generalvikariat, wenngleich erfolglos, seine Einflusssphäre bei der Unterbringung eines Sexualstraftäters auf öffentliche Einrichtungen auszudehnen. Mit der Bitte, dem ,unglücklichen Mitbruder zu helfen, wurde an die Solidarität unter Priestern appelliert. In der DDR rekurrierten Klinikärzte und Angehörige der Staatsanwaltschaft angesichts überfüllter psychiatrischer Einrichtungen hingegen auf Doneisers vermeintliche (unheilbare) Geisteskrankheit und aktivierten damit die degenerationstheoretische Deutung von ,abweichender Sexualität als Wesensmerkmal, um eine Abschiebung in die Bundesrepublik zu ermöglichen. Weil der Schutz potenzieller Opfer weder im Erzbistum noch auf DDR-Seite eine Rolle spielte, konnte die Behauptung einer sicheren Unterbringung Doneisers gerade deshalb als Scharnier zwischen West und Ost fungieren, weil das Vorhandensein einer solchen Möglichkeit von keiner Seite überprüft wurde, sondern lediglich eine Sprachhülse darstellte.

\section{Psychiatrische Expertise und seelsorgerische Betreuung: Zweiteilung im Umgang mit Sexualstraftätern?}

Obgleich auch das Erzbistum Paderborn die Bindung zu Doneiser kappen wollte, fand dieser, wie andere Sexualstraftäter auch, zunächst Aufnahme im Franziskanerkloster Wiedenbrück. Das Kloster wurde vom Erzbistum Paderborn „als Zufluchtsort Emeritierter und als Korrektionshaus strafbarer Geistlicher $[\ldots]^{4}{ }^{47}$ genutzt. In einem späteren Brief des Klostervorstehers an den Generalvikar wird eine weitere Dimension des Klosteraufenthalts erkennbar: das "[B]üssen“.88

Zwei Tage nach seiner Ankunft in der Bundesrepublik zog Doneiser seinen Laisierungsantrag zurück. ${ }^{89}$ Dem Generalvikar teilte er mit, dass er nur unter Zwang unterschrieben habe, weil dies „allein der Weg in die Freiheit gewesen

\footnotetext{
87 Internes Schreiben, Generalvikariat, 1.6.1968, EBAP: A36.10.oo9, 1968-1969(70).

88 Johanneshaus an Droste, 10.1.1969, EBAP: SA 124.

89 Doneiser an Jaeger, 29.5.1967, EBAP: SA 124.
} 
sei.“90 In einem weiteren Brief an Jaeger „als meinen geistlichen Vater“ verortete er sein Handeln im theologischen Diskursfeld der Sünde, Gnade und Vergebung: „Ich bin mir der Schwere meiner Schuld bewußt [...]. Ich bereue meine mehrmaligen Verfehlungen aus tiefster Seele, und bin bereit auch kirchliche Buße zu leisten. [...] Bitte gewähren Sie mir die Gnade, den Rest meiner Lebensjahre im Schatten des Altares zu verbringen. ${ }^{“ 91}$ Grundlegend ist hier das kanonische Recht, das sexuellen Missbrauch Minderjähriger als Verstoß gegen das sechste Gebot wertet. Die eigentliche Straftat war also der „Verstoß gegen das Enthaltsamkeitsgelübde von Klerikern. ${ }^{\text {92 }}$ In schweren Fällen sah das Kirchenrecht die Laisierung vor. ${ }^{93}$

Indem das Erzbistum jedoch offizielle Maßnahmen gegen Doneiser unterlassen hatte, eröffnete es, nach dessen Weigerung, den Laisierungsantrag zu unterschreiben, Handlungsräume für den Täter. Die vom Erzbistum zugesagte Kontrolle und Versorgung musste nun innerhalb der Institution und in Kooperation mit Doneiser erfolgen.

Vor diesem Hintergrund griff das Erzbistum, wie auch andere Diözesen seit spätestens Ende der 1920er Jahre, auf eine psychiatrische Expertise zurück. ${ }^{94}$ Den Kontakt zu einem Psychiater stellte einer der zuvor angeschriebenen „Anstaltspfarrer" her. ${ }^{95}$ Der Brief des Generalvikars an Dr. Jahn vom Westfälischen Landeskrankenhaus Marsberg offenbart das Fehlen eines kirchlichen Konzeptes zum Umgang mit Sexualstraftätern: „Für uns stellt sich nun die Frage: was sollen wir mit dem Herrn Confrater Doneiser anfangen? [...] Können wir es verantworten, ihn frei herumgehen zu lassen? Können wir es noch einmal versuchen, ihn in einem Heim, wo er einigermaßen beaufsichtigt werden kann, als Priester auftreten zu lassen? Müssen wir in seinem Interesse

\footnotetext{
$90 \quad$ Aktennotiz Droste, 12.6.1967, EBAP: SA 124.

91 Doneiser an Jaeger, 29.5.1967, EBAP: SA 124.

92 Stefan Ernst, ,Ein Kleriker, der sich auf andere Weise gegen das sechste Gebot des Dekalogs verfehlt'. Anmerkungen und Anfragen aus moraltheologischer Sicht, in: Heribert Hallermann u.a. (Hrsg.), Der Strafanspruch der Kirche in Fällen von sexuellem Missbrauch. Würzburg 2012, 185-209, hier 187.

93 Charles J. Scicluna, Ein Überblick über die Entwicklung des kanonischen Rechts im Bereich des sexuellen Missbrauchs durch Kleriker, in: Hallermann u.a. (Hrsg.), Strafanspruch, $325^{-3} 35$, hier 330.

94 EBAP: SA 339. Siehe zu den Niederlanden: Marit E. Monteiro, Discretion and Expertise. Exploring the Role of Roman Catholic Psychiatrists in the Approach and Response of the Roman Catholic Governing Bodies to the Sexual Abuse, in: Wim Deetman/Nel Draijer/ Pieter Kalbfleisch (Hrsg.), Sexual Abuse of Minors in the Roman Catholic Church, Bd. 2, 2013, 38-83, einzusehen unter https://mobile.repository.ubn.ru.nl/bitstream/ handle/2066/134155/134155.pdf?sequence=1, zuletzt aufgerufen am 4.3.2021.

Droste an Jahn, 17.6.1969, EBAP: SA 124.
} 
und vor allem auch im Interesse der Kirche - Ihr würde man ja im Falle einer neuen Straffälligkeit den Vorwurf machen, den Sachverhalt gekannt und trotzdem nicht genügend vorgesorgt zu haben - für eine echte Sicherungsverwahrung sorgen? Wo gibt es eventuell ein Haus, in dem wir ihn unterbringen könnten?" ${ }^{96}$ Die Verhinderung weiterer sexueller Gewalt war ausweislich dieses Schreibens geboten, um Doneiser vor sich selbst und vor allem auch die Kirche zu schützen. Erst vor diesem Hintergrund nahm das Erzbistum die psychiatrische Deutung sexueller Gewalt an Kindern als Geisteskrankheit, von der Priester ebenso wie andere Personen befallen sein können, auf. ${ }^{97}$ Die von Doneiser ausgehende Gefahr für Minderjährige spielte in den Überlegungen des Erzbistums eine marginale Rolle. Auch die Option, Doneiser wieder in der Seelsorge einzusetzen, wurde noch in Betracht gezogen, sofern die psychiatrische Expertise eine Wiederholungsgefahr ausschloss.

Jahn sagte zu, Doneiser zu begutachten und bot an, ihn im Haus von Ordensschwestern unterzubringen, um so seine Aufnahme als Psychiatriepatient zu vermeiden. ${ }^{98}$ Auf ein Honorar verzichtete Jahn. Im Unterschied zur Unterbringung Doneisers im Bezirkskrankenhaus Uchtspringe war diese Begutachtung vollständig in das katholische Milieu des Erzbistums eingebettet, obwohl staatliche Institutionen genutzt wurden.

Jahn stellte eine Wiederholungsgefahr fest, sprach sich aber gegen eine Laisierung aus: „Die Gefahr neuer Straftaten wäre dann in jedem Falle größer und würde auch die Kirche - da die Zeitungen dann doch immer von einem laisierten Priester berichten würden - irgendwie treffen." ${ }^{\text {"99 Der Psychiater }}$ nahm hier eine Perspektive ein, die einen möglichen Imageschaden für die Kirche hervorhob, die Schutzbedürftigkeit potenzieller Betroffener jedoch nicht als handlungsleitend ansah. Mit seiner Argumentation folgte Jahn einer seit den 195oer Jahren unter Psychiatern, die der katholischen Kirche nahestanden, häufigen Einstellung, eine Weiterbeschäftigung als Seelsorger abzulehnen, zugleich aber in einer beruflichen Untätigkeit die Gefahr weiterer Straftaten zu sehen. Das Generalvikariat schloss aufgrund des Gutachtens nun eine Laisierung Doneisers aus und Doneiser kehrte in das Kloster Wiedenbrück zurück.

Zwar wurde im Briefverkehr des Erzbistums mehrfach der Wunsch geäußert, „dem bedauernswerten Mitbruder zu helfen. ${ }^{“ 100}$ Allerdings beschränkte

\footnotetext{
$96 \quad$ Ebd.

97 Monteiro, Discretion, 39.

98 Droste an Doneiser, 11.6.1967, EBAP: SA 124.

99 Psychiatrisches Gutachten, LKH Marsberg, 3.8.1967, EBAP: SA 124.

100 Droste an Johanneshaus, 18.9.1967, EBAP: SA 124.
} 
sich die Hilfe des Erzbistums entgegen des zeitgenössischen Ratschlags von Psychiater:innen, dass eine Beschäftigungslosigkeit zu vermeiden sei, auf die Unterbringung. Dieser Umstand wurde auch vom Klostervorsteher in Wiedenbrück als unzureichend empfunden, weshalb er sich mit der Frage an das Generalvikariat wandte, wann Doneiser das Kloster verlassen könne, da die Beschäftigungslosigkeit für den noch leistungsfähigen Doneiser immer mehr zum Problem würde. ${ }^{101}$

Rückblickend legt die Glaubenskongregation dar, dass im Zeitraum nach dem Zweiten Vatikanischen Konzil bis zum Inkrafttreten der Neuregelung des kanonischen Rechts, also zwischen 1965 und 1983, unter den Bischöfen eine Haltung vorherrschte, in Fällen von sexuellem Missbrauch von Minderjährigen auf eine "pastorale Herangehensweise ${ }^{102}$, bzw. ein therapeutisches Konzept zu setzten und „Heilen“ gegenüber Strafen zu bevorzugen. Aus diesem Grund, so die Glaubenskongregation, wurden nur selten kirchenrechtliche Verfahren eingeleitet. Am Beispiel der Niederlande hat die Kirchenhistorikerin Marit Monteiro gezeigt, dass in den 196oer Jahren nur wenige Psychiater:innen, die zudem der katholischen Kirche nahestanden, in Einzelfällen davon ausgingen, dass mit Unterstützung durch die katholische Gemeinschaft und bei guter Anpassung des Täters eine Wiederholungsgefahr minimiert werden könne. ${ }^{103}$ Die Integration psychiatrischer Expertise ging also keineswegs mit einer therapeutischen Absicht einher. Das psychiatrische Fachurteil diente der Entscheidung über die weitere Berufslaufbahn von Sexualstraftätern. Das Beispiel Doneisers erhellt zudem, dass eine pastorale Begleitung des Straftäters kaum stattfand. Vielmehr zielten die Maßnahmen des Erzbistums, Versetzung und Abschottung, auf die Vermeidung eines Skandals.

\section{Medizinische und monetäre Einhegung des Täters}

Schließlich zog der 57-jährige Doneiser im April 1969, knapp zwei Jahre nach seiner Ankunft in der Bundesrepublik, in das neuerrichtete Haus für "alte und kranke Priester"104 der Johannesbrüder: Haus Johanneshöhe in FeldkirchenHüllenberg. Am Bau des Hauses hatten sich die nordrheinwestfälischen

\footnotetext{
101 Franziskanerkloster Wiedenbrück an Droste, 10.1.1969, EBAP: SA 124.

102 Die Normen des Motu proprio „Sacramentorum Sanctitatis Tutela“. Geschichtliche Einführung, zusammengestellt von der Kongregation für die Glaubenslehre, einzusehen unter http://www.vatican.va/resources/resources_introd-storica_ge.html, zuletzt aufgerufen am 3.1.2021.

103 Monteiro, Discretion, 43.

104 Aktenvermerk Droste, 22.5.1969, EBAP: SA 124.
} 
Diözesen finanziell beteiligt und konnten dafür ein bis zwei Wohnungen mit Bistumsangehörigen belegen. ${ }^{105}$ Für eine psychiatrische Behandlung der Bewohner stand offenbar ein niedergelassener Psychiater zur Verfügung. ${ }^{106}$

Als Doneiser im Folgejahr eine Reise nach Wien plante, trat das durch dieVertuschung der Tat begründete gegenseitige Abhängigkeitsverhältnis zwischen Doneiser und dem Erzbistum klar hervor. Das Erzbistum wollte die Reise "an gewisse Bedingungen " ${ }^{107}$ knüpfen, war aber, da keine kirchenrechtlichen Sanktionen erfolgt waren, nicht in der Lage, Doneiser konkrete Vorschriften zu erteilen. Daher griff Generalvikar Droste auf eine bereits zuvor ergangene Anfrage des früheren Gutachters von Doneiser, des Psychiaters Jahn, zurück. Dieser prüfte im Landeskrankenhaus Marsberg mittlerweile einen Arzneistoff zur Triebhemmung, der zwei Jahre später unter dem Namen „Androcur“ auf den Markt kommen sollte. ${ }^{108}$ Generalvikar Dorste betonte, dass dieser Arznei-

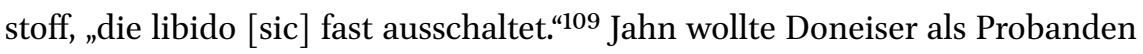
in seine Studie aufnehmen. Der Generalvikar forderte Doneiser deshalb auf, sich mit Jahn in Verbindung zu setzen, damit, wie es Droste in verharmlosenden Worten formulierte, so „eventuell unliebsamen Vorkommnissen bei einer Ferienreise ${ }^{\text {"110 }}$ vorgebeugt werden könne. Dieser Vorschlag steht in der Tradition von Kastrationen, die früher in Einzelfällen der katholischen Kirche nahestehende Psychiater empfohlen hatten. ${ }^{111}$ Indem das Generalvikariat Doneiser dazu riet, sich mit Jahn in Verbindung zu setzen, wurde die Verantwortung für den ehemaligen Straftäter erneut an die Psychiatrie delegiert. Ethisch fragwürdig war sowohl das Ansinnen des Arztes, da das Pharmaunternehmen verurteilte Straftäter als Probanden ausgeschlossen hatte, ${ }^{112}$ als auch die Kopplung der Reise an die Einnahme eines Versuchsstoffs seitens des Erzbistums. Ob Doneiser der Aufforderung nachkam, lässt sich der Überlieferung nicht entnehmen, allerdings ist eine Notiz mit Doneisers Namen und einer Wiener Adresse erhalten. ${ }^{13}$ Sicher ist, dass Doneiser 1972 nach Portugal reiste. Weitere sieben Jahre später verstarb Doneiser 1979 im Haus Johanneshöhe. Für den Aufenthalt im Kloster Wiedenbrück und im Haus Johanneshöhe

105 Generalvikar Köln an Generalvikare in Essen, Mainz, Münster, Osnabrück, Paderborn, Trier, 20.3.1968, Droste an Johanneshaus, 15.1.1969; Johanneshaus an Droste, 30.9.1967, EBAP:SA 124.

106 Siehe den Aufsatz von Bernhard Frings in diesem Band.

107 Droste an Johanneshaus, 12.5.1970, EBAP: SA 124.

108 Protokoll, 12.3.1971, Schering Archiv: B9-42.

109 Droste an Johanneshaus, 12.5.1970, EBAP: SA 124.

110 Droste an Johanneshaus, 12.5.70, EBAP: SA 124.

111 Monteiro, Discretion, 46.

112 Bericht, 1.10.1970, Schering Archiv: S1 229.

113 Notiz, o.D., EBAP: SA 124. 
sowie für einen monatlichen Betrag zur freien Verfügung kam seit der Einreise Doneisers in die Bundesrepublik im Mai 1967 größtenteils das Erzbistum auf. Der Umgang mit Doneiser erfolgte trotz des immer wieder aufscheinenden Mitgefühls mit einem Täter auch oder vor allem unter der Prämisse, diesen einzuhegen und das Image der Institution Kirche zu wahren.

\section{Zusammenfassung}

Dieser Beitrag untersuchte Bedingungen, die es Klerikern erleichterten, sexuelle Gewalt gegen Minderjährige auszuüben. Eine mikrohistorische Herangehensweise zeigt, dass der dabei zu berücksichtigende Personenkreis sich in der hier betrachteten Zeit von Mitte der 195oer bis Mitte der 197oer Jahre nicht auf jene kirchlichen Leitungspersonen beschränkte, die nach dem bisherigen Stand der Forschung zumeist Kenntnis von sexueller Gewalt gegenüber Minderjährigen besaßen: (Erz-)Bischof und Generalvikar sowie in diesem Fall der Erzbischöfliche Kommissar in Magdeburg. Diese Menschen trugen die personelle und juristische Verantwortung, übten sie jedoch nur unzureichend aus. So unterblieben eine Unterstützung Doneisers als er im Vorfeld der ersten Tat über Probleme klagte, eine Betreuung und Kontrolle, nachdem er versetzt wurde, kirchenrechtliche Schritte sowie die Fürsorge gegenüber den Betroffenen.

Erkennbar wird ebenso die Teilhabe und oft auch situative Unterstützung des Täters durch einen Kreis von Klerikern und Laien, darunter Ansprechpartner in Altenheimen, Psychiatrien, Wohlfahrtseinrichtungen etc., von denen ein Teil in den Diensten des Bistums stand, während bei anderen die Bindung persönlicher Natur war. Diese Gruppe beschreibt das jeweils spezifische katholische Milieu, das Kenntnis von und Einfluss auf den Umgang mit Tätern besaß. Psychiater:innen, „Anstaltspfarrer“ etc. erfüllten dabei gerade nicht ihre dienstlichen Aufgaben, sondern stellten ihre institutionellen Ressourcen in den Dienst des Erzbistums. Ihre Zustimmung oder Ablehnung der vom Generalvikariat erwogenen Maßnahmen beeinflusste den kirchlichen Umgang mit Sexualstraftätern und konturiert damit die Reichweite der kirchlichen Einflusssphäre. Zu diesem Kreis zählen auch die Mitglieder der Pfarrgemeinden, in denen Beschuldigte tätig waren. Aufgrund der Quellenlage musste in diesem Beitrag eine Analyse dieses wichtigen Feldes unterbleiben.

Das Handeln von Psychiater:innen und Staatsanwaltschaft wurde am Beispiel der DDR der 195oer und 196oer Jahre untersucht. Eine Analyse ihrer Äußerungen lässt keine Bindungen an das katholische Milieu erkennen, wie es für Jurist:innen und Psychiater:innen im Westteil des Erzbistums vielfach angenommen werden kann und am Beispiel des Psychiaters Jahn auch gezeigt 
wurde. Dies zeigt, dass in den 196oer Jahren auch außerhalb des katholischen Milieus ein Klima der Akzeptanz von sexueller Gewalt existierte, das von einer Negierung der Betroffenenperspektive gekennzeichnet war. Dies gilt bis in die 1970er Jahre hinein nicht nur für die DDR, sondern ebenso für die Bundesrepublik. ${ }^{14}$ Seitens der Justiz wurde nur ein Bruchteil sexueller Gewalt gegen Minderjährige geahndet und im Fall von Doneiser Betroffenen nur eingeschränkt Glauben geschenkt. Die Psychiatrie, von juristischer Seite als Expertin aufgefordert, über das Gefährdungspotenzial von Tätern zu entscheiden, folgte im betrachteten Zeitraum mehrheitlich einem degenerationstheoretischen Ansatz, der Ursachen von sexueller Gewalt im ,Wesen' der Täter verortete. Sie ging daher von einer dauerhaften Gefährdung durch den Täter aus, die lediglich in Einzelfällen durch Anpassung, Kastration oder Medikamente unterdrückt werden konnte. Trotz des Argumentes einer dauerhaften Gefährdung waren aber beide Institutionen, Justiz und Psychiatrie, bereit, die weitere Verantwortung für Doneiser abzustreifen und der Kirche zu überlassen.

Eine mikrohistorische Analyse erhellt also, warum die titelgebende Frage „Können wir es verantworten, ihn frei herumgehen zu lassen“ - vom Erzbistum Paderborn auf der einen und Justiz und Psychiatrie auf der anderen Seite zwar unterschiedlich beantwortet wurde, Vertreter:innen aller drei Institutionen aber miteinander kooperierten. Aufgrund von milieuübergreifenden Schweigeregimen und aufgrund der Unterstützung von Tätern innerhalb des katholischen Milieus konnte sich das Erzbistum Paderborn im hier betrachteten Untersuchungszeitraum, flankiert von psychiatrischer Expertise, auf Buße, Vergebung und Neuanfang beschränken. Kleriker, die sexuelle Gewalt gegen Minderjährige ausgeübt hatten, wurden innerhalb des Erzbistums, aber auch innerhalb des katholischen Milieus, vielfach nicht als Straftäter, sondern als bedauernswerte Mitbrüder und Sünder wahrgenommen. Die Folgen sexueller Gewalt für Minderjährige, ihre Erfahrungen und der Schutz von Betroffenen erlangten hingegen kaum Betrachtung. Diese Sichtweise trug dazu bei, einer Vertuschung von Straftaten Vorschub zu leisten, den Täter zu schützen, eine persönliche wie innerinstitutionelle Auseinandersetzung zu verhindern und solchermaßen den Handlungsraum von Tätern nicht zu begrenzen.

114 Felicitas Söhner/Heiner Fangerau, Medizinhistorische Perspektive auf die Wandlung des Verständnisses von sexualisierter Gewalt gegen Kinder, in: Alexandra Retkowski/Mart Busche/Elisabeth Tuider (Hrsg.), Handbuch Sexualisierte Gewalt \& pädagogische Arbeit. Weinheim 2018, 81-89, hier 81ff. 